\title{
Propiedades Psicométricas de la Escala Multidimensional de Apoyo Social Percibido en Estudiantes Universitarios Chilenos
}

\author{
Psychometric Properties of the Multidimensional Scale of Perceived Social Support \\ in Chilean University Students
}

\author{
Yaranay López-Angulo ${ }^{1}$, M. Victoria Pérez-Villalobos ${ }^{2}$, Ana Bernardo Gutiérrez $^{3}$, \\ Rubia Cobo Rendón ${ }^{4}$, Fabiola Sáez Delgado ${ }^{5}$ y Alejandro Díaz-Mujica ${ }^{6}$
}

\begin{abstract}
Resumen
La Escala Multidimensional de Apoyo Social Percibido fue diseñada para evaluar la percepción de apoyo social desde tres fuentes: familia, amigos y otros significativos. La presente investigación tuvo como objetivo principal determinar las propiedades psicométricas del MSPSS cuestionario a través de una aplicación a 1975 estudiantes que cursaban el primer y segundo año de sus carreras en Universidades del Sur de Chile (55.031\% mujeres), con edades comprendidas entre 17 y 25 años $(M=19.293 ; D E=1.349)$. Los resultados indicaron un adecuado ajuste del modelo de segundo orden considerando tres dimensiones del apoyo (familia, amigos y otros significativos). Los coeficientes de consistencia interna para las dimensiones de la escala fueron, $\alpha=.903$ (Familia), $\alpha=.928$ (Amigos) y $\alpha=.864$ (otros significativos). Se concluye que el cuestionario es un instrumento válido y confiable para evaluar el apoyo social percibido en estudiantes universitarios chilenos y además posee equivalencia de medida en función del sexo.
\end{abstract}

Palabras clave: apoyo social percibido, confiabilidad, validez, estudiantes universitarios

\begin{abstract}
The Multidimensional Scale of Perceived Social Support was designed to assess perceived social support according to three sources: family, friends and other significant ones. The main objective of this research was to determine the psychometric properties of this questionnaire through its application to 1975 students in the first and second year of their careers at Universities in Southern Chile (55.031\% women), aged between 17 and 25 years-old $(\mathrm{M}=19.293 ; \mathrm{SD}=1.349)$. The results indicated adequate adjustment of the second order model considering three dimensions of support (family, friends and other significant ones). The internal consistency coefficients for the dimensions of the scale were, $\alpha=.903$ (Family), $\alpha=.928$ (Friends) and $\alpha=.864$ (other significant ones). It is concluded that the questionnaire is a valid and reliable instrument to evaluate this construct in chilean university students and it also has equivalence of measure across gender.
\end{abstract}

Keywords: perceived social support, reliability, validity, university students

Agradecimientos: Este artículo contó con financiamiento CONICYT. Proyecto Fondecyt Regular 1161502: "Modelo explicativo de la permanencia y el abandono de los estudios universitarios, basado en procesos cognitivo motivacionales" 2016-2020 y en el

marco del proyecto de investigación "Modelo explicativo del ajuste académico y la intención de abandono en estudiantes universitarios" para optar al grado de doctor en Psicología de la Universidad de Concepción, Chile. Cuenta con financiamiento de Becas Doctorado Nacional CONICYT Folio 21170795.

\footnotetext{
${ }^{1}$ Doctora(c) en Psicología, Facultad de Ciencias Sociales y Comunicaciones, Universidad Santo Tomás, Concepción, Chile. Tel.: +56986769901. Correo: yaralopez@udec.cl (Autor de correspondencia)

${ }^{2}$ Doctora en Psicología, Departamento de Psicología, Universidad de Concepción, Chile. Tel.: +56412203955. Correo: marperez@udec.cl

${ }^{3}$ Doctora en Psicología, Departamento de Psicología, Universidad de Oviedo, España. Tel.: +985 1095 41. Correo: bernardoana@uniovi.es

4 Doctora(c) en Psicología, Departamento de Psicología, Universidad de Concepción, Chile. Tel.: +56985112942. Correo: rubiacobo@udec.cl

${ }^{5}$ Doctora en Psicología, Universidad Católica de la Santísima Concepción, Concepción, Chile. Tel.: +56412345913. Correo: fsaez@ ucsc.cl

${ }^{6}$ Doctor en Psicología, Departamento de Psicología, Universidad de Concepción, Chile. Tel.: +56412203955. Correo: adiazm@udec.cl

Revista Iberoamericana de Diagnóstico y Evaluación - e Avaliação Psicológica. RIDEP · No58 · Vol.1 · 127-140 · 2021

ISSN: 1135-3848 print /2183-6051online
} 


\section{Introducción}

El ingreso en la universidad demanda a los estudiantes desafíos personales importantes en el plano familiar, académico, social y emocional (Baker \& Siryk, 1986; Credé \& Niehorster, 2012). Con frecuencia, los estudiantes en los primeros años de sus carreras presentan dificultades para adaptarse al nuevo contexto, las cuales pueden verse reflejadas en el bajo desempeño en los estudios, disminución de la satisfacción académica, altos niveles de estrés, depresión y ansiedad; poniendo en riesgo la permanencia en la universidad (Brandy, Penckofer, Solari-Twadell, \& Velsor-Friedrich, 2015; Conley, Kirsch, Dickson, \& Bryant, 2014; Ibrahim, Kelly, Adams, \& Glazebrook, 2013). La incidencia del abandono es sobre todo elevada en estudiantes de primer año; la explicación deriva en las dificultades que los estudiantes de nuevo ingreso presentan en la transición y adaptación académica en términos de autonomía y madurez en la universidad (Araújo, Gomes, Almeida, \& Núñez, 2019).

Uno de los recursos que los estudiantes universitarios utilizan para hacerle frente a estas dificultades es el apoyo social. Por ejemplo, algunos estudiantes deben abandonar el domicilio familiar ya que la universidad a la que acuden no está cerca de su residencia y pierden el soporte parental. Para tales estudiantes, es importante crear nuevas redes de apoyo social entre sus compañeros y profesores que apoyen sus vivencias sociales y emocionales. Percibir que se recibe un trato justo y honesto de parte de los compañeros, profesores y demás miembros de la institución, disfrutar de la atmósfera del campus y sus actividades, así como de buenas relaciones personales contribuye a la permanencia de los estudiantes en la universidad (Esteban, Bernardo, Tuero, Cerezo, \& Núñez 2016; Motl, Multon, \& Zhao, 2018). Estudios recientes muestran la importancia que tiene para el estudiante percibir apoyo social; por lo cual es considerado un predictor valioso para la adaptación y permanencia en este nuevo contexto (Akanni \& Oduaran, 2018; Meng, Huang, Hou, \& Fan, 2015; Rodríguez, Tinajero, \& Páramo, 2017; Salami, 2011).

El apoyo social es considerado un constructo multidimensional. En tal sentido existen diversas formas de conceptualizarlo; por ejemplo puede entenderse como las provisiones instrumentales y/o expresivas, reales o percibidas, que ofrece la comunidad, redes sociales y personas de confianza (Lin, Dean, \& Ensel, 1986), o como la percepción individual que cada persona tenga de 6 tipos de provisiones sociales: alianza confiable, orientación, apego, integración social, refuerzo de valía y oportunidad de cuidar (Weiss, 1974). También puede ser entendido como la percepción de las personas a través de 5 dimensiones: dirección (apoyo dado o recibido), disposición (disponible o se está utilizando el apoyo), descripción/evaluación (apoyo social descrito o simplemente evaluado), contenido (emocional, instrumental, de información o la valoración de soporte) y red (familiares, amigos, maestros, etc.) (Tardy, 1985), y como el número personas a la que se puede recurrir y el grado de satisfacción con el apoyo disponible (Sarason, Levine, Basham, \& Sarason, 1983).

Para la medición del apoyo social existen varios instrumentos: (1) Social Support Questionnaire es un instrumento compuesto por dos escalas independientes, la primera mide número total de personas que brindan apoyo y la segunda mide la satisfacción con el apoyo recibido (Sarason et al., 1983); (2) The Duke University Functional Social Support, mide el apoyo social funcional en base a la dimensión de apoyo de un confidente y apoyo afectivo (Broadhead, Gehlbach, De Gruy, \& Kaplan, 1988); el apoyo social afectivo cubre el apoyo de las personas que se preocupan y de las personas que dan amor y afecto; el apoyo social de confianza cubre oportunidades para hablar sobre problemas personales, financieros o relacionados con el trabajo y la participación en actividades con otros; (3) Escala de Provisiones Sociales (Cutrona \& Russell, 1987) mide diferentes funciones del apoyo a través de seis dimensiones: alianza confiable, orientación, apego, integración social, refuerzo de valía y oportunidad de cuidar y (4) Multidimensional Scale of Perceived Social Support (MSPSS) creado por Zimet, Dahlem, Zimet, and Farley (1988). El MSPSS fue creado para medir percepción de apoyo social proveniente de tres fuentes o dimensiones: familia, amigos y otros significativos. 
Este último instrumento, tiene como fortalezas: 1) la evaluación de la percepción de apoyo emocional recibido, por tanto, se centra en aspectos subjetivos; 2) precisa y explora el apoyo social proveniente de las tres fuentes de apoyo social más relevantes de cualquier ser humano familia, amigos-otros significativos-; 3) tiene una presentación clara, breve y de respuesta rápida (Zimet et al., 1988).

El MSPSS es un instrumento de alto reconocimiento para la evaluación del apoyo social percibido, cuenta con estudios de validez y adaptaciones en diferentes países y con diversas muestras. En América, específicamente en Estados Unidos se ha utilizado en muestras de mujeres embarazadas, adolescentes y residentes pediátricos (Zimet, Powell, Farley, Werkman, \& Berkoff, 1990); adultos mayores con ansiedad generalizada y sin patologías (Stanley, Beck, \& Zebb, 1998); personas con esquizofrenia o trastornos afectivos (Cecil, Stanley, Carrion, \& Swann, 1995); adultos mayores con trastornos de ansiedad y sin patologías diagnosticadas (Stanley et al., 1998); mujeres árabes inmigrantes (Aroian, Templin, \& Ramaswamy, 2010). En muestra de adultos mayores (Pinto, Lara, Espinoza, \& Montoya, 2014) y pacientes diabéticos tipo II (Ortiz-Parada \& Baeza-Rivera, 2011) en Chile y con trabajadores (Gabardo-Martins, Ferreira, \& Valentini, 2017) en Brasil.

En Asia, se han desarrollado estudios con muestras de jóvenes y adolescentes (Guan, Seng, Hway Ann, \& Hui, 2015) y en estudiantes de medicina (Ng, Siddiq, Aida, Zainal, \& Koh, 2010) en Malasia; con estudiantes universitarios (Guan et al., 2015), en pacientes que consumen metadona (Zhou et al., 2015) y en adolescentes (Kee-Lee, 2000) en China; con pacientes de psiquiatría, cirugía y normales (Eker, Arkar, \& Yaldiz, 2000) y en estudiantes universitarios (Duru, 2007) en Turquía; con estudiantes de medicina y pacientes psiquiátricos (Wongpakaran, Wongpakaran, \& Ruktrakul, 2011) en Tailandia; con población adulta con esquizofrenia (Vaingankar, Abdin, \& Chong, 2012) en Singapur; con mujeres embarazadas (Akhtar et al., 2010) en Pakistán. En África, con jóvenes con síntomas depresivos y de ansiedad (Bruwer, Emsley, Kidd, Lochner, \& Seedat, 2008) en Sudáfrica.
En Europa, con mujeres con hirsutismo y estudiantes de enfermería (Ekbäck, Benzein, Lindberg, \& Årestedt, 2013) en Suecia; mujeres en período posparto (Denis, Callahan, \& Bouvard, 2015) en Francia; con estudiantes universitarios (Adamczyk, 2013) en Polonia; con prejubilados inscritos en los programas universitarios (LópezRamos, Fernández-Muñoz, Navarro-Pardo, \& Murphy, 2017) en España.

El análisis de los estudios mencionados permite confirmar que la validez de constructo del MSPSS es muy estable, en la mayoría se confirma la estructura factorial del instrumento original tres factores- lo cual es válido por los índices de ajustes obtenidos. Sin embargo, investigaciones confirman una estructura distinta, en China Hong Kong, con muestra de adolescentes encuentran una solución de dos factores (Kee-Lee, 2000) y en Pakistán (Akhtar et al., 2010) confirman un solo factor general. Estas inconsistencias respecto a la estructura factorial de la escala pueden estar dadas por diferentes razones: (1) diferencias en cuanto a la percepción de apoyo en función de cada cultura, de modo que, estudios realizados en culturas muy colectivas donde la distinción entre familia y otros significativos se hace muy difusa (Qadir, Khalid, Haqqani, Medhin, \& Huma, 2013); (2) estudios con falta de rigor en el análisis de los datos puesto que utilizan métodos de rotación ortogonal, lo cual no corresponde dado que teóricamente los factores del MSPSS están relacionados; utilizan una muestra muy pequeña o utilizan como método de extracción de factores el análisis de componentes principales, entre otros elementos que producen conclusiones inexactas (Arechabala \& Miranda, 2002; Cobb \& Xie, 2015); (3) imprecisiones respecto a la calidad del proceso de traducción a otros idiomas; esta ha sido considerada una de las limitaciones a las adaptaciones del MSPSS.

Aunque el MSPSS se ha utilizado en variadas culturas y poblaciones, no se ha encontrado estudios sobre la utilidad en estudiantes universitarios de habla hispana, por tanto, es valioso tener una medida válida y confiable para el contexto de la Educación Superior, que permita evaluar las fuentes de apoyo social. La presente investigación es relevante, en tanto, proporciona información psicométrica sobre el uso de esta escala en el contexto de la Educación Superior 
chilena haciendo posible que otros estudios identifiquen la fuente de apoyo que los estudiantes perciben como más significativa, contribuyendo así al desarrollo de intervenciones efectivas dirigidas al incremento del apoyo social percibido. En consecuencia, el objetivo de esta investigación es determinar las propiedades psicométricas del MSPSS en estudiantes universitarios chilenos.

Otro aspecto relevante son las diferencias en el modelo según el sexo. En la literatura se han mostrado diferencias significativas respecto a la percepción de apoyo social entre hombres y mujeres, siendo los hombres quienes presentan menor percepción de apoyo (Tayfur \& Ulupinar, 2016); sin embargo, no se han encontrado evidencias en cuanto a la invariancia factorial del modelo para hombres y mujeres.

A partir de los antecedentes presentados se plantean las siguientes hipótesis alternativas: $\mathrm{H}_{1}$ : El MSPSS posee una estructura unidimensional, es decir, todos los ítems conforman un solo factor; $\mathrm{H}_{2}$ : El MSPSS está compuesto por dos factores de primer orden; $\mathrm{H}_{3}$ : El MSPSS está compuesto por tres factores de primer orden; $\mathrm{H}_{4}$ : El MSPSS está compuesto por tres factores de primer orden y un factor de segundo orden y $\mathrm{H}_{5}$ : El MSPSS presentará valores de ajuste adecuados de invarianza factorial en función del sexo.

\section{Método}

La presente investigación corresponde a un diseño de tipo instrumental (Ato, López, \& Benavente, 2013), orientada al análisis de las propiedades psicométricas del MSPSS (Zimet et al., 1988), a la evaluación de su estructura factorial en una muestra de estudiantes universitarios chilenos; y al análisis de la invarianza factorial en función del sexo.

\section{Participantes}

La población fueron estudiantes universitarios de la región del Bio-Bio, para el 2019, esta región contaba con un total de 125.425 estudiantes distribuidos en 9 universidades (SIES, 2017). Se encuestaron a 1975 estudiantes de siete universidades del sur de Chile. Los estudiantes pertenecían a diferentes carreras de las áreas de Ciencias Básicas, Ingeniería, Educación, Salud,
Ciencias Sociales, Humanidades, Arte, Arquitectura, Administración y Comercio, de estos 888 hombres (44.962\%) y 1087 mujeres (55.031\%). Sus edades estuvieron comprendidas entre 17 y 25 años $(M=19.293 ; D E=1.349)$ de estos el $89 \%$ de los estudiantes se encontraban cursando el primer año de la universidad y el $11 \%$ restante, corresponde a estudiantes de segundo año. En la Tabla 1, se describen los participantes según el área científica a la cual pertenece su carrera para la Organización para la Cooperación y el Desarrollo Económico (OCDE).

Tabla 1. Descripción de los participantes según sexo y área de conocimiento

\begin{tabular}{lccc}
\hline Área científica según la & \multicolumn{2}{c}{ Sexo } & \multirow{2}{*}{ n } \\
\cline { 2 - 3 } OCDE & Hombres & Mujeres & \\
\hline Ingeniería y Tecnología & 404 & 255 & 659 \\
Ciencias Agrícolas & 41 & 63 & 104 \\
Ciencias Sociales & 268 & 437 & 705 \\
Humanidades & 11 & 14 & 25 \\
Ciencias Médicas y de la & 133 & 303 & 436 \\
Salud & 31 & 15 & 46 \\
Ciencias Naturales & 888 & 1087 & 1975 \\
Total & & & \\
\hline
\end{tabular}

\section{Instrumento}

El MSPSS fue creado para medir percepción de apoyo social proveniente de tres fuentes: familia, amigos y otros significativos. Es un instrumento de auto-informe con una escala de respuesta de tipo Likert y va desde 1=muy en desacuerdo hasta 7=muy de acuerdo. Está compuesto por 12 ítems organizados en 3 dimensiones de 4 ítems cada una: (1) Familia "Mi familia me da la ayuda y apoyo emocional que requiero", Amigos "Puedo conversar de mis problemas con mis amigos" y (3) Otros significativos "Cuando necesito ayuda, sé que hay alguien que me puede apoyar". En cuanto a la fiabilidad de las dimensiones reportada por sus autores esta fue heterogénea ( $\alpha=.85$ familia, $\alpha=.87$ amigos y .91 otros significativos) (Zimet et al., 1988). En esta investigación se utilizó la versión en idioma español de Arechabala y Miranda (2002). Este estudio confirmó una estructura factorial de dos dimensiones y la fiabilidad fue de $(\alpha=.86$ familia $\mathrm{y}$ otros significativos y $\alpha=.88$ amigos). 


\section{Procedimiento}

Los datos fueron recogidos en el marco de aplicaciones de un proyecto de investigación más amplio. Los coordinadores del proyecto contactaron e informaron a las autoridades de las universidades participantes (decanos y jefes de carrera) las características del estudio; quienes aceptaron participar, completaron la autorización correspondiente y presentaron disponibilidad de fechas para las aplicaciones. Los encuestadores accedieron a las salas de clases, invitaron a los estudiantes a participar explicando sobre qué trataba el estudio. Los estudiantes dispuestos firmaron el consentimiento informado y procedieron a responder el instrumento.

La validez del instrumento se realizó mediante análisis factoriales confirmatorios de manera exploratoria, para evaluar la estructura subyacente del MSPSS y comparar sus ajustes (Bollen, 1989). Se utilizó el test multivariado de Mardia para el análisis de la normalidad multivariada. La estimación de los parámetros se realizó con el estimador MLR (Maximum Likelihood Robust); éste permite tratar las variables como continuas (escala ordinal de 7 puntos) y no requiere el cumplimiento de los supuestos de normalidad, puesto que se considera lo suficientemente robusto como para detectar un efecto cuando este realmente existe (Lloret-Segura, Ferreres-Traver, Hernández-Baeza, \& Tomás-Marco, 2014). Se utilizó el software MPlus 7.11 (Muthén \& Muthén, 1998-2017).

Los índices considerados para evaluar el ajuste del modelo fueron: $\chi^{2}$ Chi Cuadrado, RMSEA (Root Mean Square Error of Approximation), SRMR (Standardised Root Mean Square Residual), CFI (Comparative fit index) y TLI (Índice de Tucker-Lewis). Los criterios de análisis considerados fueron los siguientes: índices de Chi-cuadrado, se espera encontrar valores no significativos. La raíz cuadrada media de error de aproximación (RMSEA) considera un buen ajuste cuando existen valores entre.050-.080 y un intervalo de confianza al $90 \%$ (I.C.) entre .000 - .050; el índice de ajuste comparativo (CFI) $\mathrm{y}$ índice de Tucker-Lewis se esperan valores superiores a .950 (Brown, 2014; Geiser, 2012; Hair, Black, Babin, \& Anderson, 2014). La consistencia interna del instrumento fue estimada mediante el coeficiente omega, para el alfa de
Cronbach se utilizó el software libre JASP v. 0.8.3.1 (JASP Team, 2017); se esperan encontrar valores superiores a .700 (Luján-Tangarife \& Cardona-Arias, 2015). Se utilizó el programa estadístico SPSS 21 (IBM, 2011) para el análisis de estadísticos descriptivos de la muestra normativa chilena.

Para la evaluación de la invarianza factorial, el marco de referencia fue el análisis factorial confirmatorio (AFC) basado en la estructura de medias y covarianzas (MACS) (Meredith, 1993). Para ello, se compararon jerárquicamente siete modelos anidados comenzando con el esquema configural que sirvió como punto de partida. Considerando que el instrumento tiene un esquema compuesto por tres factores de primer orden y uno de segundo orden, se examinaron los cuatro niveles de invarianza recomendados utilizando el sexo como característica sociodemográfica de agrupación, pero incorporando tanto las variables observadas como las latentes. Específicamente, los modelos evaluados fueron:

(1) Modelo configural (modelo 1): modelo sin restricciones en el que los parámetros fueron estimados libremente. Este esquema sirvió como referencia para los demás y se empleó para establecer el primer nivel de invarianza conocida como configural.

(2) Modelo con cargas factoriales de primer orden restringidas (modelo 2): en este caso se impuso la condición de que las cargas factoriales de primer orden fueran iguales entre varones y hembras. A este tipo de invarianza se le define como métrica o débil.

(3) Modelo con cargas factoriales de segundo orden restringidas (modelo 3): el siguiente nivel implicó, además de las limitaciones anteriores, la igualdad de los coeficientes de regresión de las variables latentes.

(4) Modelo con interceptos de primer orden restringidos (modelo 4): en esta oportunidad se agregó otro impedimento que consistió en forzar los interceptos de los elementos o ítems para que fuesen iguales entre adolescentes masculinos y femeninos, lo cual, de comprobarse, aportaría evidencias a favor de la invarianza escalar o fuerte.

(5) Modelo con interceptos de segundo orden restringidos (modelo 5): además de la condición anterior, se impuso en este modelo la igualdad 
Tabla 2. Estadísticos descriptivos de los ítems y las dimensiones de la Escala Multidimensional de Apoyo Social Percibido

\begin{tabular}{|c|c|c|c|c|c|c|}
\hline \multirow{2}{*}{ Ítems } & \multirow{2}{*}{$\begin{array}{c}\text { Media } \\
\text { Estadístico }\end{array}$} & \multirow{2}{*}{$\begin{array}{l}\text { Desviación } \\
\text { Estadístico }\end{array}$} & \multicolumn{2}{|c|}{ Asimetría } & \multicolumn{2}{|c|}{ Curtosis } \\
\hline & & & Estadístico & Desv. Error & Estadístico & Desv. Error \\
\hline A1 & 5.590 & 1.401 & -1.121 & .055 & 0.814 & .110 \\
\hline $\mathrm{A} 2$ & 5.580 & 1.510 & -1.127 & .055 & 0.629 & .110 \\
\hline A3 & 5.800 & 1.507 & -1.327 & .055 & 1.065 & .110 \\
\hline A4 & 6.130 & 1.287 & -1.743 & .055 & 2.779 & .110 \\
\hline A5 & 5.890 & 1.463 & -1.476 & .055 & 1.664 & .110 \\
\hline A6 & 5.650 & 1.500 & -1.237 & .055 & 1.006 & .110 \\
\hline A7 & 5.710 & 1.431 & -1.311 & .055 & 1.432 & .110 \\
\hline A8 & 5.360 & 1.696 & -0.975 & .055 & 0.078 & .110 \\
\hline A9 & 5.690 & 1.461 & -1.265 & .055 & 1.164 & .110 \\
\hline A10 & 5.900 & 1.323 & -1.411 & .055 & 1.795 & .110 \\
\hline A11 & 5.290 & 1.792 & -0.927 & .055 & -0.149 & .110 \\
\hline A12 & 5.680 & 1.405 & -1.198 & .055 & 1.197 & .110 \\
\hline
\end{tabular}

entre los participantes de los interceptos vinculados con los factores de segundo orden o medias.

(6) Modelo con varianza residual restringida (modelo 6): en este esquema se restringieron todos los parámetros previos más los residuales asociados a los reactivos. De verificarse esta suposición, se estaría estableciendo el primer nivel de invarianza estricta.

(7) Modelo con perturbaciones restringidas (modelo 7): por último, se fijó la varianza de las variables latentes o perturbaciones para que fueran iguales en ambos grupos.

El procesamiento y análisis de los datos fue ejecutado con el programa R-Studio (versión 1.3.1073), específicamente con los paquetes Lavaan, Semtools, Semplot, Mvn, Mvnoutlier, entre otros.

\section{Resultados}

En la Tabla 2 se presentan los estadísticos descriptivos para los 12 ítems del MSPSS. Todos los ítems obtuvieron puntajes ligeramente superiores al punto medio de la escala, siendo el ítem 4 el que tuvo mayor grado de acuerdo. En cuanto al análisis univariado de los datos, la asimetría y la curtosis muestran que la mayoría de los ítems estuvieron dentro del rango esperado teóricamente de -2 y +2 (Bandalos \& Finney, 2010) solo el ítem 4, referido a "Tengo la seguridad que mi familia trata de apoyarme" presentó una curtosis ligeramente superior, indicando que las respuestas de los participantes se agrupan en mayor medida en las puntuaciones intermedias de la escala. El test multivariado de Mardia (1970), indicó que la distribución de los datos no es similar a la de una distribución normal $(p<.001)$.

\section{Propiedades Psicométricas del MSPSS}

Con el objetivo de evaluar la estructura factorial del MSPSS se emplearon análisis factoriales confirmatorios de manera exploratoria (Bollen, 1989; Schmitt, 2011) estos análisis permiten modelar distintas estructuras factoriales en coherencia con la evidencia de los resultados obtenidos en otras investigaciones. En la Tabla 3 se presentan los resultados del ajuste obtenido para los diferentes modelos probados.

Inicialmente, se realizó el análisis del ajuste de modelo considerando una distribución unifactorial de los ítems, en base a los resultados obtenidos por (Akhtar et al., 2010). En el caso de los resultados de esta investigación, el análisis factorial mostró bajo ajuste de los datos, puesto que los índices obtenidos no cumplieron con los criterios adecuados sugeridos en la literatura RMSEA $\leq .070(\mathrm{Hu} \&$ Bentler, 1999; Steiger, 2007); CFI y TLI >.950 y SRMR <.080 (Hair et al., 2014); lo que indicó que el MSPSS no ajusta en una estructura de una sola dimensión.

El primer y segundo modelo, evaluaron la estructura factorial de primer orden compuesta por dos factores, la distribución escogida corresponde a los hallazgos reportados por Kee-Lee (2000) y Arechabala and Miranda (2002) índices de ajuste fuera del rango de lo esperado teóricamente, indicando un ajuste pobre del modelo. Se presentan un valor significativo del test ChiCuadrado, el RMSEA es superior al rango recomendado .050 - .080, esperándose valores inferiores a este criterio y el índice de ajuste comparativo no se encuentra dentro del rango $>.950$ (Geiser, 2012). Estos resultados indicaron que el MSPSS no presenta una estructura de 
Tabla 3. Índices de ajuste para modelos de la estructura factorial de la Escala Multidimensional de Apoyo Social Percibido

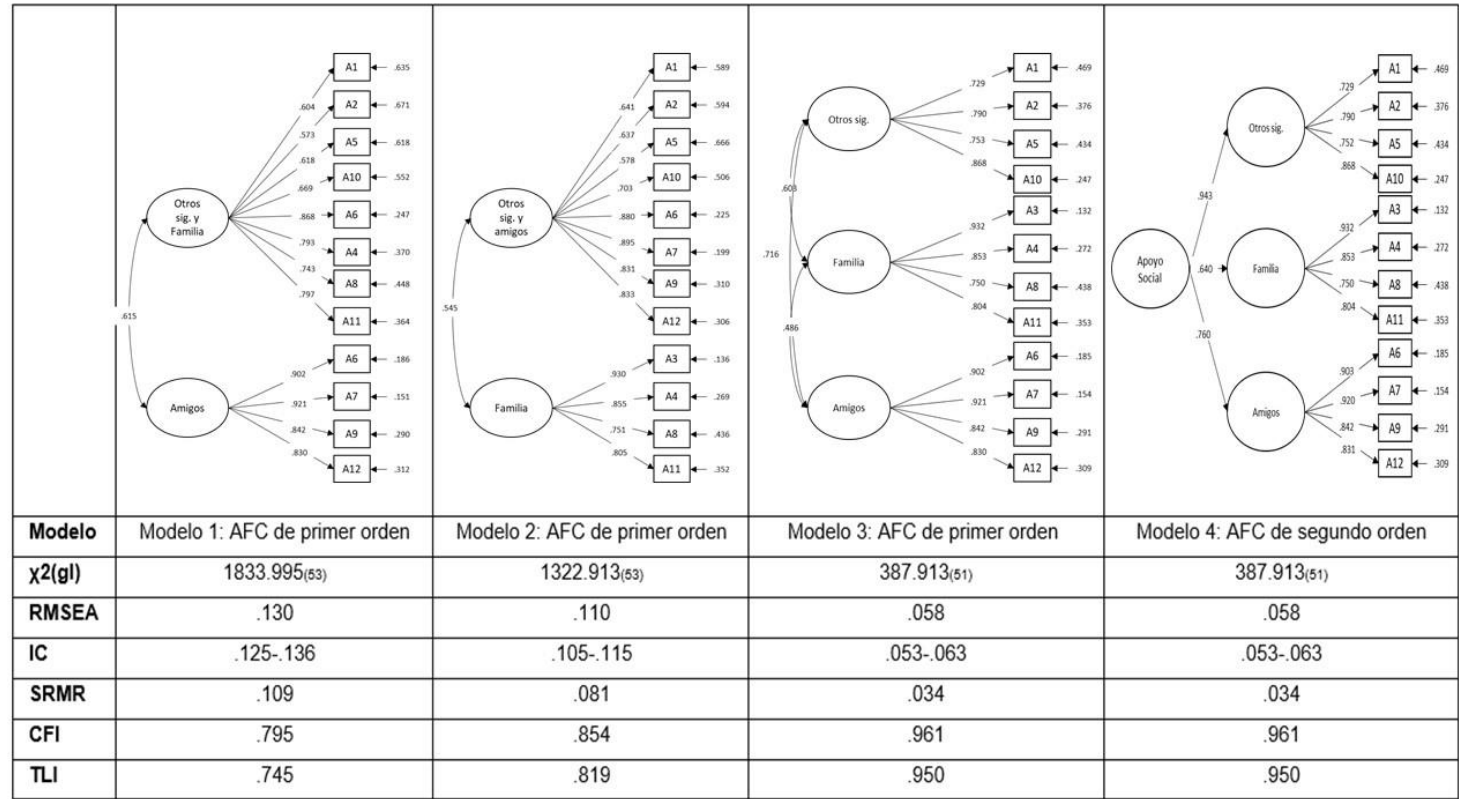

Abreviaturas: $\mathrm{OS}=$ otros significativos, $\mathrm{F}=$ familia, $\mathrm{A}=$ amigos, $\mathrm{AFC}=$ Análisis Factorial Confirmatorio

primer orden con factores fusionados en ninguna de sus posibles combinaciones.

Los modelos 3 y 4, presentan un modelo recursivo de tres dimensiones que constituyen un factor general. Estas propuestas de estructura factorial indican un ajuste a los datos significativamente superior a los modelos anteriores; ambos coinciden en sus índices de ajuste. Esta similitud estadística es habitual encontrarla cuando hay niveles equivalentes de ajuste en modelos con factores correlacionados (modelo 3) y factores de segundo orden (modelo 4); dado que se presenta una estructura factorial con todos los factores relacionados entre sí, lo que supone indirectamente la existencia de un factor latente, que explica al mismo tiempo esas relaciones (Bentler, 2005).

Como se observa en la Tabla 3, el modelo 4 de segundo orden de tres factores presenta un adecuado ajuste a los datos y es coherente con el planteamiento teórico del MSPSS; por lo tanto, se concluye que el MSPSS está conformado por tres factores específicos, proveniente de la evaluación de tres fuentes de apoyo, que permiten dar cuenta del constructo de apoyo social percibido.

En tal sentido, el análisis de validez considerado para analizar es el correspondiente al modelo 4. En la Tabla 4 se reportan las cargas factoriales obtenidas de los tres factores propuestos para el análisis; todos presentaron cargas significativas en el factor de segundo orden. En general, los ítems presentaron cargas adecuadas en cada factor, confirmándose las dimensiones de (a) Familia, (b) Amigos y (c) Otros significativos como dimensiones específicas que pueden ser agrupadas en un factor general (Ver Tabla 4).

Tabla 4. Cargas factoriales para la Escala Multidimensional de Apoyo Social Percibido

\begin{tabular}{lccc}
\hline Ítem & Familia & Amigos & Otros significativos \\
\hline A1 & $.713(.016)$ & & \\
A2 & $.786(.013)$ & & \\
A5 & $.748(.014)$ & & \\
A10 & $.860(.010)$ & & \\
A3 & & $.931(.007)$ & \\
A4 & & $.851(.011)$ & \\
A8 & & $.736(.014)$ & $.902(.008)$ \\
A11 & & $.797(.011)$ & $.920(.008)$ \\
A6 & & $.841(.010)$ \\
A7 & & $.834(.011)$ \\
A9 & & \\
A12 & &
\end{tabular}


Tabla 5. Correlación ítem-dimensión de la Escala Multidimensional de Apoyo Social Percibido

\begin{tabular}{|c|c|c|c|}
\hline Ítems & $\mathrm{F}$ & A & OS \\
\hline A3 Mi familia me da la ayuda y apoyo emocional que requiero & $.909^{* *}$ & & \\
\hline A4 Tengo la seguridad que mi familia trata de apoyarme & $.855^{* *}$ & & \\
\hline A8 Mi familia me ayuda a tomar decisiones & $.861^{* *}$ & & \\
\hline A11 Puedo conversar de mis problemas con mi familia & $.892^{* *}$ & & \\
\hline A6 Puedo conversar de mis problemas con mis amigos & & $.921^{* *}$ & \\
\hline A7 Puedo contar con mis amigos cuando tengo problemas & & $.928^{* *}$ & \\
\hline A9 Cuando tengo alegrías o penas puedo compartirlas con mis amigos & & $.895^{* *}$ & \\
\hline A12 Tengo la seguridad de que mis amigos tratan de apoyarme & & $.882^{* *}$ & \\
\hline A1 Cuando necesito ayuda, sé que hay alguien que me puede apoyar & & & $.805^{* *}$ \\
\hline A2 Hay una persona que me ofrece consuelo cuando lo necesito & & & $.868^{* *}$ \\
\hline A5 Hay una persona que se interesa por lo que yo siento & & & $.821^{* *}$ \\
\hline A10 Cuando tengo dificultades tengo a alguien que me apoya & & & $.875^{* *}$ \\
\hline
\end{tabular}

Nota. $\mathrm{N}=1975 ; * * p<.01$

Tabla 6. Coeficientes de consistencia interna para la Escala Multidimensional de Apoyo Social Percibido

\begin{tabular}{lccc}
\hline Factores & No. de ítems & Alpha de Cronbach & Omega \\
\hline Familia & $3,4,8,11$ & .903 & .904 \\
Amigos & $6,7,9,12$ & .928 & .929 \\
Otro significativo & $1,2,5,10$ & .864 & .866 \\
General & Todos & .922 & .922 \\
\hline
\end{tabular}

Tabla 7. Invarianza de la Escala Multidimensional de Apoyo Social Percibido según sexo

\begin{tabular}{lccccccc}
\hline Medida & Modelo 1 & Modelo 2 & Modelo 3 & Modelo 4 & Modelo 5 & Modelo 6 & Modelo 7 \\
\hline $\mathrm{X}^{2 \mathrm{a}}$ & $452.090^{* * * *}$ & $469.369^{* * *}$ & $475.490^{* * *}$ & $509.602^{* * *}$ & $544.811^{* * * *}$ & $568.467^{* * * *}$ & $572.205^{* * * *}$ \\
$\mathrm{gl}$ & 102 & 111 & 113 & 122 & 125 & 137 & 141 \\
CFI & .960 & .959 & .959 & .956 & .952 & .951 & .951 \\
RMSEA & .059 & .057 & .057 & .057 & .058 & .056 & .056 \\
ICB RMSEA & $.055-.066$ & $.053-.061$ & $.053-.061$ & $.053-.061$ & $.054-.062$ & $.053-.060$ & $.052-.059$ \\
SRMR & .036 & .038 & .040 & .042 & .050 & .051 & .055 \\
Comparación & NA & Modelo 1 & Modelo 2 & Modelo 3 & Modelo 4 & Modelo 5 & Modelo 6 \\
$\Delta$ gl & NA & 9 & 2 & 9 & 3 & 12 & 4 \\
$\Delta \mathrm{X}^{2 \mathrm{~b}}$ & NA & $13.87^{\dagger}$ & $5.30^{\dagger}$ & $31.52^{* * *}$ & $52.67^{* * *}$ & $34.08^{* * *}$ & $6.04^{\dagger}$ \\
$\Delta$ CFI & NA & .001 & .000 & .003 & .004 & .001 & .000 \\
$\Delta$ RMSEA & NA & $.002^{\ddagger}$ & .000 & .000 & .001 & $.002^{\ddagger}$ & .000 \\
$\Delta$ SRMR & NA & .002 & .002 & .002 & .008 & .001 & .004 \\
\hline
\end{tabular}

Modelo 1: modelo sin restricciones para evaluar la invarianza configural. Modelo 2: modelo con cargas factoriales iguales para evaluar la invarianza métrica de primer orden. Modelo 3: modelo con coeficientes de regresión iguales para evaluar la invarianza métrica de segundo orden. Modelo 4: modelo con interceptos iguales para evaluar invarianza escalar de primer orden. Modelo 5: modelo con medias iguales para evaluar invarianza escalar de segundo orden. Modelo 6: modelo con varianzas residuales iguales para evaluar invarianza estricta de primer orden. Modelo 7: modelo con perturbaciones iguales para evaluar invarianza estricta de segundo orden. a Se muestra el estadístico chi-cuadrado escalado de Satorra-Bentler. ${ }^{b}$ La diferencia en los chi-cuadrados corresponde a la ajustada o robusta. Nomenclatura: $\Delta$ : símbolo delta. Representa la diferencia en los índices de los modelos comparados. ${ }^{\dagger}$ No significativo. ${ }^{* * *}$ Significativo para .001. ${ }^{\dagger}$ Disminución del RMSEA en vez de aumento.

En cuanto a las correlaciones de los ítems del MSPSS, en la Tabla 5 se observa que todos los ítems tuvieron correlaciones significativas y de magnitud alta con la dimensión a donde corresponden, lo que refleja niveles de asociación adecuados en todos los ítems del instrumento con respecto a sus dimensiones.

\section{Confiabilidad}

La confiabilidad se calculó mediante el índice de alfa de Cronbach ( $\alpha$ ) y el coeficiente Omega $(\omega)$ de McDonalds (1978). Se elige este último test porque diferentes investigadores sugieren usarlo para garantizar mayor precisión en el cálculo de la fiabilidad, en tanto, su cálculo no depende del número de ítems ni de las opciones de respuesta como el alfa, sino de las cargas factoriales (Ventura-León \& CaychoRodríguez, 2017). Basado en estos análisis, los factores "Familia" y "Amigos" y "Otros significativos" obtuvieron valores de confiabilidad adecuados a lo esperado teóricamente. Estos coeficientes sugieren que este instrumento es una medida fiable de apoyo social percibido para estudiantes universitarios, ver Tabla 6.

\section{Invarianza Factorial}

Los hallazgos sustentan la invarianza factorial de la escala MSPSS. Como se muestra 
en la Tabla 7 los cambios en el CFI, RMSEA y SRMR fueron en todo momento inferiores a los puntos de corte sugeridos. No obstante, la diferencia ajustada en los estadísticos chicuadrado fue significativa cuando se compararon los modelos 3, 4 y 5 con los esquemas predecesores (Tabla 7). Esto podría reflejar violaciones a la equivalencia métrica, escalar y residual; sin embargo, probablemente éstas sean discrepancias provocadas por el tamaño muestral de esta investigación. En consecuencia, no hay razones de peso para rechazar la invarianza factorial del instrumento psicométrico evaluado.

\section{Discusión}

El presente estudio tuvo como objetivo determinar las propiedades psicométricas del MSPSS, en una muestra de estudiantes universitarios chilenos, con énfasis en la evaluación de la estructura factorial subyacente para corroborar si ésta se ajustaba al modelo teórico propuesto por los autores creadores del instrumento.

Con este objetivo se probaron las primeras cuatro hipótesis establecidas en esta investigación relacionadas con la estructura factorial del MSPSS. Los resultados permitieron rechazar la $\mathrm{H}_{1}, \mathrm{H}_{2}$ y $\mathrm{H}_{3}$; confirmando el cumplimiento de la $\mathrm{H}_{4}$, la cual precisa que el MSPSS está compuesto por tres factores de primer orden y un factor de segundo orden. Este resultado muestra una estructura jerárquica compuesta por un factor general y tres factores específicos, coherente con lo propuesto por los creadores del instrumento (Zimet et al., 1988). De este modo, los resultados agregan evidencia que apoya una configuración jerárquica del cuestionario MSPSS, compuesta por tres factores latentes de primer orden (familia, amigos y otros significativos), más un factor latente de segundo orden. Esta configuración teórica es coherente con los hallazgos de López-Ramos et al. (2017). Los resultados presentados en este artículo difieren de aquellos encontrados por Akhtar et al. (2010) y Kee-Lee (2000), puesto que en este caso la propuesta de estructura de un factor o dos no presentaron adecuado ajuste. Este hallazgo apoya la idea sobre la inconsistencia en la estructura de esta escala; lo cual puede deberse a otros factores como por ejemplo las diferencias culturales (Qadir et al., 2013).

Para comprobar la última hipótesis $\left(\mathrm{H}_{5}\right)$ relacionada con la invarianza factorial en función del sexo, se evaluaron siete modelos anidados a través de la invarianza de medida o equivalencia de un modelo factorial de medida entre diferentes grupos, en este caso hombres y mujeres. Para determinar la equivalencia de la escala se cotejó el ajuste de cada modelo al compararlo con un esquema anterior con menos imposiciones, utilizando para ello la prueba de la diferencia en los chi-cuadrado. Debido a las características del instrumento y la ausencia de normalidad, el método de estimación robusto adoptado fue el de ML con estadístico escalado (Satorra \& Bentler, 1994), estrategia identificada en paquetes como Mplus y Lavaan bajo los acrónimos MLR o MLM. Un problema vinculado al test antes mencionado y al uso de estos estimadores es que la diferencia en los estadísticos no sigue una distribución chi-cuadrado (Bentler, 2005), de manera que el resultado reportado en esta publicación corresponde a la diferencia corregida propuesta por varios investigadores (Bryant \& Satorra, 2012; Satorra \& Bentler, 2010).

Asimismo, se analizó el CFI, RMSEA y el SRMR. La utilización adicional de estos indicadores se justifica debido a la sensibilidad del estadístico chi-cuadrado cuando la muestra es grande, hecho que llevaría al rechazo de la hipótesis nula, aunque las discrepancias observadas no tengan relevancia práctica (Cheung \& Rensvold, 2002; Little, 1997; Marsh, Hey, Roche, \& Perry, 1997). Con relación a estas medidas, se ha propuesto que una disminución en el CFI mayor que .01 sería un indicio de no equivalencia, en tanto que aumentos en el RMSEA o en el SRMR mayores que .015 reflejarían una violación de la invarianza factorial (Cheung \& Rensvold, 2002). Los análisis de invarianza factorial de medida, permitieron confirmar la $\mathrm{H}_{5}$, mostrándose que el MSPSS presentó valores de ajuste adecuados de invarianza factorial en función del sexo.

Estos resultados tienen implicaciones psicométricas, educativas y clínicas. En cuanto a las implicancias psicométricas, se puede decir que existe evidencia empírica a favor del modelo 
teórico propuesto por Zimet et al. (1988), lo cual apoya la validez de constructo de este instrumento. Las implicaciones en el área clínica y educativa apuntan a la posibilidad de tener un instrumento que permita delimitar la fuente del apoyo percibido más relevante y menos relevante desde la percepción de los estudiantes.

En el ámbito educativo, dada la importancia del apoyo social percibido en el rendimiento académico (González \& Guadalupe, 2017) es pertinente evaluar específicamente la fuente de apoyo social tal y como es percibida por el estudiante. Aunque hay valiosas propuestas para la medición del apoyo social (Cuéllar-Flores \& Dresch, 2012; Gallardo-Peralta \& Gálvez-Nieto, 2018), prevalece la necesidad de implementar acciones educativas intencionadas que incidan donde radica la dificultad. Este cuestionario MSPSS- es lo suficientemente sensible como para arrojar resultados fiables sobre la fuente de apoyo específica a intervenir. Por ejemplo, en jornadas de acogida, participación en grupos académicos, deportivos y otros (Fernandez-Castañon et al., 2017; Peña, 2017).

En el caso de la estabilidad de las respuestas, el MSPSS presentó buenos índices de fiabilidad a través de los coeficientes de Omega y Cronbach, como tendencia, los índices oscilaron entre .929 y .864 , siendo adecuada la consistencia interna para cada una de sus dimensiones.

En síntesis, los resultados obtenidos permiten confirmar la estructura propuesta por los autores, referida a la naturaleza multidimensional del constructo; así como la posibilidad de ser interpretados a través de un puntaje total. Además, la evaluación de la invarianza factorial mostró que el MSPSS tiene valores de ajuste adecuados inalterables tanto para hombres como para mujeres. También se identificaron adecuados niveles de fiabilidad en las respuestas de los participantes, confirmando que esta escala es un instrumento adecuado para ser empleado en el contexto de la educación universitaria chilena.

\section{Referencias}

Adamczyk, K. (2013). Development and validation of the Polish-language version of the Multidimensional Scale of Perceived Social Support (MSPSS). Revue
Internationale de Psychologie Sociale, 26(4), 25-48.

https://www.cairn.info/revue-internationalede-psychologiesociale-2013-2014-page2025.htm.

Akanni, A. A., \& Oduaran, C. A. (2018). Perceived social support and life satisfaction among freshmen: Mediating roles of academic self-efficacy and academic adjustment. Journal of Psychology in Africa, 28(2), 89-93. doi:10.1080/14330237.2018.1454582

Akhtar, A., Rahman, A., Husain, M., Chaudhry, I. B., Duddu, V., \& Husain, N. (2010). Multidimensional scale of perceived social support: psychometric properties in a South Asian population. Journal of Obstetrics and Gynaecology Research, 36(4), 845-851. doi:10.1111/j.1447-0756.2010.01204.x

Araújo, A. M., Gomes, C. M. A., Almeida, L. S., \& Núñez, J. C. (2019). A latent profile analysis of first-year university students' academic expectations. Anales de Psicología/Annals of Psychology, 35(1), 5867. doi:10.1080/03075079.2016.1196350

Arechabala, M. C., \& Miranda, C. (2002). Validación de una escala de apoyo social percibido en un grupo de adultos mayores adscritos a un programa de hipertensión de la región metropolitana. Ciencia y Enfermería, $8(1), 49-55$. doi:10.4067/S0717-95532002000100007

Aroian, K., Templin, T. N., \& Ramaswamy, V. (2010). Adaptation and psychometric evaluation of the Multidimensional Scale of Perceived Social Support for Arab immigrant women. Health Care for Women International, $\quad 31(2), \quad$ 153-169. doi:10.1080/07399330903052145

Ato, M., López, J. J., \& Benavente, A. (2013). Un sistema de clasificación de los diseños de investigación en psicología. Anales de Psicología, 29(3), 1038-1059. doi:10.6018/analesps.29.3.178511

Baker, R. W., \& Siryk, B. (1986). Exploratory intervention with a scale measuring adjustment to college. Journal of Counseling Psychology, 33(1), 31-38. doi:10.1037/00220167.33.1.31

Bandalos, D. L., \& Finney, S. J. (2010). Factor analysis: Exploratory and confirmatory. In 
The reviewer's guide to quantitative methods in the social sciences (pp. 110-134): Routledge.

Bentler, P. M. (2005). EQS 6.1 for Windows. Structural equations program manual. Encino, CA: Multivariate Software, Inc., 16, 78-117.

Bollen, K. A. (1989). Structural equations with latent variables Wiley. New york.

Brandy, J. M., Penckofer, S., Solari-Twadell, P. A., \& Velsor-Friedrich, B. (2015). Factors predictive of depression in first-year college students. Journal of Psychosocial Nursing Mental Health Services, 53(2), 38-44. doi:10.3928 / 02793695-20150126-03

Broadhead, W., Gehlbach, S. H., De Gruy, F. V., \& Kaplan, B. H. (1988). The Duke-UNC Functional Social Support Questionnaire: Measurement of social support in family medicine patients. Medical Care, 709-723. https://www.jstor.org/stable/3765493.

Brown, T. A. (2014). Confirmatory factor analysis for applied research (Second ed.). New York: Guilford Publications.

Bruwer, B., Emsley, R., Kidd, M., Lochner, C., \& Seedat, S. (2008). Psychometric properties of the Multidimensional Scale of Perceived Social Support in youth. Comprehensive Psychiatry, 49(2), 195-201. doi:10.1016/j.comppsych.2007.09.002

Bryant, F. B., \& Satorra, A. (2012). Principles and practice of scaled difference chi-square testing. Structural equation modeling: A Multidisciplinary Journal, 19(3), 372-398. doi:10.1080/10705511.2012.687671.

Cecil, H., Stanley, M. A., Carrion, P. G., \& Swann, A. (1995). Psychometric properties of the MSPSS and NOS in psychiatric outpatients. Journal of Clinical Psychology, 51(5), 593-602. doi:10.1002/10974679(199509)51:5<593::AID-

JCLP2270510503>3.0.CO;2-W

Cheung, G. W., \& Rensvold, R. B. (2002). Evaluating goodness-of-fit indexes for testing measurement invariance. Structural Equation Modeling: A Multidisciplinary Journal, 9(2), 233-255. doi:10.1207/S15328007SEM0902_5

Cobb, C. L., \& Xie, D. (2015). Structure of the multidimensional scale of perceived social support for undocumented Hispanic immigrants. Hispanic Journal of Behavioral Sciences, 37(2), 274-281. doi:10.1177/0739986315577894

Conley, C. S., Kirsch, A. C., Dickson, D. A., \& Bryant, F. B. (2014). Negotiating the transition to college: Developmental trajectories and gender differences in psychological functioning, cognitive-affective strategies, and social well-being. Emerging Adulthood, 2(3), 195-210. doi:10.1177/2167696814521808

Credé, M., \& Niehorster, S. (2012). Adjustment to college as measured by the student adaptation to college questionnaire: A quantitative review of its structure and relationships with correlates and consequences. Educational Psychology Review, 24(1), 133-165. doi:10.1007/s10648-011-9184-5

Cuéllar-Flores, I., \& Dresch, V. (2012). Validación del cuestionario de Apoyo Social Funcional Duke-UNK-11 en personas cuidadoras. Revista Iberoamericana de Diagnóstico y Evaluación - e Avaliação Psicológica, 2(34), 89-101. http://www.redalyc.org/articulo.oa?id=459645 438004.

Cutrona, C. E., \& Russell, D. W. (1987). The provisions of social relationships and adaptation to stress. Advances in Personal Relationships, $\quad$ 1(1), 3767.https://www.researchgate.net/publication/2 71507385.

Denis, A., Callahan, S., \& Bouvard, M. (2015). Evaluation of the French version of the multidimensional scale of perceived social support during the postpartum period. Maternal and Child Health Journal, 19(6), 1245-1251. doi:10.1007/s10995-014-1630-9

Duru, E. (2007). Re-examination of the psychometric characteristics of the multidimensional scale of perceived social support among Turkish university students. Social Behavior and Personality: An International Journal, 35(4), 443-452. doi:10.2224/sbp.2007.35.4.443

Ekbäck, M., Benzein, E., Lindberg, M., \& Årestedt, K. (2013). The Swedish version of the multidimensional scale of perceived social support (MSPSS)-a psychometric evaluation study in women with hirsutism and nursing 
students. Health and Quality of Life Outcomes, 11(168), 1-9. http://www.hqlo.com/content/11/11/168.

Eker, D., Arkar, H., \& Yaldiz, H. (2000). Generality of support sources and psychometric properties of a Scale Of Perceived Social Support in Turkey. Social Psychiatry and Psychiatric Epidemiology, 35(5), 228-233.

https://link.springer.com/content/pdf/210.100 7/s001270050232.pdf.

Esteban, M., Bernardo, A. B., Tuero, E., Cerezo, R., \& Núñez , J. C. (2016). El contexto sí importa: identificación de relaciones entre el abandono de titulación y variables contextuales. European Journal of Education and Psychology Psychology, 9(2), 79-88. doi:10.1016/j.ejeps.2015.06.001

Fernandez-Castañon, A. C., Bernardoz, A. B., Esteban, M., Tuero, E., Carbajal, R., \& Núñez, J. C. (2017). Influencia en el abandono universitario de variables relacionales y sociales. Revista de Estudios y Investigación en Psicología y Educación(12), 46-49. doi:10.17979/reipe.2017.0.12.2531

Gabardo-Martins, L. M. D., Ferreira, M. C., \& Valentini, F. (2017). Propriedades psicométricas da escala multidimensional de suporte social percebido. Trends in Psychology / Temas em Psicologia, 25(4), 1873-1883. doi:10.9788/TP2017.4-18Pt

Gallardo-Peralta, L. P., \& Gálvez-Nieto, J. L. (2018). Validación del cuestionario de apoyo social comunitario en personas mayores chilenas. Revista Iberoamericana de Diagnóstico y Evaluación - e Avaliação Psicológica, 47(49), 47-57. doi:10.21865/RIDEP49.4.04

Geiser, C. (2012). Data analysis with Mplus: Guilford Press.

González, C., \& Guadalupe, E. (2017). Factores que inciden en el rendimiento académico de los estudiantes de la Universidad Politécnica del Valle de Toluca. Revista Latinoamericana de Estudios Educativos (México), 47(1), 91108.

http://www.redalyc.org/articulo.oa?id=270504 22005.

Guan, N. C., Seng, L. H., Hway Ann, A. Y., \& Hui, K. O. (2015). Factorial validity and reliability of the Malaysian simplified Chinese version of multidimensional scale of perceived social support (MSPSS-SCV) among a group of university students. Asia Pacific Journal of Public Health, 27(2), 225231. doi:10.1177/1010539513477684

Hair, J. F., Black, W. C., Babin, B. J., \& Anderson, R. E. (2014). Multivariate data analysis: Pearson new international edition.

Hu, L. T., \& Bentler, P. M. (1999). Cutoff criteria for fit indexes in covariance structure analysis: Conventional criteria versus new alternatives. Structural Equation Modeling: A Multidisciplinary Journal, 6(1), 1-55. doi:10.1080/10705519909540118

IBM, C. (2011). IBM SPSS statistics for Windows, version 20.0. Armonk, NY: IBM Corporation.

Ibrahim, A. K., Kelly, S. J., Adams, C. E., \& Glazebrook, C. (2013). A systematic review of studies of depression prevalence in university students. Journal of Psychiatric Research, 47(3), 391-400.

doi:10.1016/j.jpsychires.2012.11.015

Kee-Lee, C. (2000). Assessing Chinese adolescents' social support: The Multidimensional Scale Of Perceived Social Support. Personality and Individual Differences, 28(2), 299-307. doi:10.1016/S0191-8869(99)00098-7

Lin, N., Dean, A., \& Ensel, W. M. (1986). Social support, life events, and depression: London: Academic Press.

Little, T. D. (1997). Mean and covariance structures (MACS) analyses of cross-cultural data: Practical and theoretical issues. Multivariate Behavioral Research, 32(1), 5376. doi:10.1207/s15327906mbr3201_3

Lloret-Segura, S., Ferreres-Traver, A., HernándezBaeza, A., \& Tomás-Marco, I. (2014). El análisis factorial exploratorio de los ítems: una guía práctica, revisada y actualizada. Anales de Psicología, 30(3), 1151-1169.

López-Ramos, Y., Fernández-Muñoz, J. J., Navarro-Pardo, E., \& Murphy, M. (2017). Confirmatory factor analysis for the multidimensional scale of perceived social support in a sample of early retirees enrolled in university programs. Clinical Gerontologist, 40(4), 241-248. 
doi:10.1080/07317115.2016.1199077

Luján-Tangarife, J., \& Cardona-Arias, J. A. (2015). Construcción y validación de escalas de medición en salud: Revisión de propiedades psicométricas. Archivos de Medicina, 11(3), 1-10. doi:10.3823/1251

Marsh, H. W., Hey, J., Roche, L. A., \& Perry, C. (1997). Structure of physical self-concept: Elite athletes and physical education students. Journal of Educational Psychology, 89(2), 369-380. doi:10.1037/0022-0663.89.2.369

Meng, H., Huang, P., Hou, N., \& Fan, J. (2015). Social self-efficacy predicts Chinese College Students' first-year transition: A four-wave longitudinal investigation. Journal of Career Assessment, 23(3), 410-426. doi:10.1177/1069072714547482

Meredith, W. (1993). Measurement invariance, factor analysis and factorial invariance. Psychometrika, 58(4), 525-543. doi:10.1007/BF02294825

Motl, T. C., Multon, K. D., \& Zhao, F. (2018). Persistence at a tribal university: Factors associated with second year enrollment. Journal of Diversity in Higher Education, 11(1), 1-37. doi:10.1037 / dhe0000034

Muthén, L. K., \& Muthén, B. O. (1998-2017). Mplus User's Guide. Eighth Edition. Los Angeles, CA: Muthén \& Muthén.

Ng, C., Siddiq, A. N., Aida, S., Zainal, N., \& Koh, O. (2010). Validation of the Malay version of the Multidimensional Scale of Perceived Social Support (MSPSS-M) among a group of medical students in Faculty of Medicine, University Malaya. Asian Journal of Psychiatry, 3(1), 3-6. doi:10.1016/j.ajp.2009.12.001

Ortiz-Parada, M., \& Baeza-Rivera, J., María. (2011). Propiedades psicométricas de una escala para medir apoyo social percibido en pacientes chilenos con diabetes tipo 2 . Universitas Psychologica, 10(1), 189-196. http://www.redalyc.org/articulo.oa?id=647192 84016.

Peña, A. (2017). ¿ La experiencia Universitaria influye en el abandono de la Universidad? INNOVA Research Journal, 2(10), 162-168. https://repositorio.uide.edu.ec/handle/37000/3 3561.
Pinto, C., Lara, R., Espinoza, E., \& Montoya, P. (2014). Propiedades psicométricas de la escala de apoyo social percibido de Zimet en personas mayores de Atención Primaria de Salud. Index de Enfermería, 23(1-2), 85-89. doi:10.4321/S1132-12962014000100018

Qadir, F., Khalid, A., Haqqani, S., Medhin, G., \& Huma, Z. (2013). The association of marital relationship and perceived social support with mental health of women in Pakistan. BMC Public Health, 13(1), 2-13. http://www.biomedcentral.com/14712458/1413/1150.

Rodríguez, M. S., Tinajero, C., \& Páramo, M. F. (2017). Pre-entry characteristics, perceived social support, adjustment and academic achievement in first-year Spanish University students: A path model. The Journal of Psychology, 151(8), 722-738. doi:10.1080/00223980.2017.1372351

Salami, S. O. (2011). Psychosocial predictors of adjustment among first year College of Education students. US-China Education Review, 8(2), 239-248. https://files.eric.ed.gov/fulltext/ED519567.pdf.

Sarason, I. G., Levine, H. M., Basham, R. B., \& Sarason, B. R. (1983). Assessing social support: The social support questionnaire. Journal of Personality and Social Psychology, 44(1), 1-56.

https://apps.dtic.mil/dtic/tr/fulltext/u52/a1004 64.pdf.

Satorra, A., \& Bentler, P. M. (1994). Corrections to test statistics and standard errors in covariance structure analysis. En Latent variables analysis: Applications for developmental research. (pp.399-419). Sage Publications, Inc.

Satorra, A., \& Bentler, P. M. (2010). Ensuring positiveness of the scaled difference chisquare test statistic. Psychometrika, 75(2), 243-248. doi:10.1007/s11336-009-9135-y

Schmitt, T. (2011). Current methodological considerations in exploratory and confirmatory factor analysis. Journal of Psychoeducational Assessment, 29(4), 304321. doi:10.1177/0734282911406653

SIES. (2017). Informe retención de 1er año de pregrado. Cohortes 2013 - 2017. División de Educación Superior, Ministerio de 
Educación., Recuperado en https://analisis.umag.cl/documentos/retencion _ler_ano_sies_2018.pdf.

Stanley, M. A., Beck, J. G., \& Zebb, B. J. (1998). Psychometric properties of the MSPSS in older adults. Aging \& Mental Health, 2(3), 186-193. doi:10.1080/13607869856669

Steiger, J. H. (2007). Understanding the limitations of global fit assessment in structural equation modeling. Personality and Individual Differences, 42(5), 893-898. doi:10.1016/j.paid.2006.09.017

Tardy, C. H. (1985). Social support measurement. American Journal of Community Psychology, 13(2), 187-202. doi:10.1007/BF00905728

Tayfur, C., \& Ulupinar, S. (2016). The Effect of Perceived Social Support on the Academic Achievement of Health College Students. Journal of Psychiatric Nursing/Psikiyatri Hemsireleri Dernegi, 7(1). doi:10.5505/phd.2016.52523

Vaingankar, J. A., Abdin, E., \& Chong, S. A. (2012). Exploratory and confirmatory factor analyses of the Multidimensional Scale of Perceived Social Support in patients with schizophrenia. Comprehensive Psychiatry, 53(3), 286-291.

doi:10.1016/j.comppsych.2011.04.005

Ventura-León, J. L., \& Caycho-Rodríguez, T. (2017). El coeficiente omega: Un método alternativo para la estimación de la confiabilidad. Revista Latinoamericana de Ciencias Sociales, Niñez y Juventud, 15(1), 625-627.

http://www.redalyc.org/articulo.oa?id=773496 27039.

Weiss, R. S. (1974). The provisions of social relationship. . Z Rubin (Ed.), Doing unto thers, 17-26.

Wongpakaran, T., Wongpakaran, N., \& Ruktrakul, R. (2011). Reliability and validity of the multidimensional scale of perceived social support (MSPSS): Thai version. Clinical Practice and Epidemiology in Mental Health, 7, 161-166. doi:10.2174 / 1745017901107010161

Zhou, K., Li, H., Wei, X., Yin, J., Liang, P., Zhang, H., . . . Li, X. (2015). Reliability and validity of the multidimensional scale of perceived social support in Chinese mainland patients with methadone maintenance treatment. Comprehensive Psychiatry, 60, 182-188.

doi:10.1016/j.comppsych.2015.03.007

Zimet, G., Dahlem, N., Zimet, S., \& Farley, G. (1988). The Multidimensional Scale of Perceived Social Support. Journal of Personality Assessment, 52(1), 30-41. doi:10.1207/s15327752jpa5201_2

Zimet, G., Powell, S., Farley, G., Werkman, S., \& Berkoff, K. (1990). Psychometric characteristics of the multidimensional scale of perceived social support. Journal of Personality Assessment, 55(3-4), 610-617. doi:10.1080/00223891.1990.9674095 